\title{
PERFIL DE APLICAÇÃO PARA RESUMOS ESTRUTURADOS (PARE)
}

\section{APPLICATION PROFILE FOR STRUCTURED ABSTRACTS (APSA)}

\author{
Paula Regina Ventura Amorim Gonçaleza \\ Brigida Maria Nogueira Cervantes ${ }^{b}$
}

\begin{abstract}
RESUMO
Introdução: O resumo representa a versão precisa, sintética e seletiva do texto do documento, portanto, nele devem estar os elementos de maior importância. Assim, é necessário que o resumo seja feito de maneira itemizada, em que o conjunto de elementos traduza claramente e com fidedignidade o conteúdo do artigo. Objetivo: Propor um perfil de aplicação de metadados para a descrição de elementos do resumo estruturado no domínio dos artigos científicos publicados em periódicos científicos eletrônicos. Metodologia: Análise exploratória com abordagem qualitativa. A pesquisa foi realizada na literatura da área de Medicina Geral e na área de Ciência da Informação. Na literatura da área médica, que já utiliza os resumos estruturados, foram pesquisados artigos nas bases de dados Medical Literature Analysis and Retrieval System Online (MEDLINE) e Literatura Latino-Americana e do Caribe em Ciências da Saúde (LILACS). $\mathrm{Na}$ área da Ciência da Informação, a pesquisa documental foi feita a partir do levantamento dos periódicos qualificados pela Capes, quadriênio 2013-2016, tendo como amostra 72 títulos na área da Ciência da Informação e os 15 primeiros títulos apresentados no ranking InCites Journal Citation Reports (JCR) e estratificados pela Capes. Como instrumentos de pesquisa utilizou-se: Extrato Capes para periódicos na área da Ciência da Informação e a ferramenta InCites Journal Citation Reports (JCR) para periódicos na área de Medicina Geral. Resultados: Apresenta a Proposição do Perfil de Aplicação para Resumos Estruturados (PARE). Conclusão: O resumo deve ser o espelho das informações contidas nos artigos e deve ser sistematicamente construído para que os leitores possam: identificar pontos relevantes de seu interesse que estejam cientificamente fundamentados; contar com uma estrutura que lhes apresente de forma concisa os resultados das pesquisas; e pesquisar por motores de busca mais precisos.
\end{abstract}

Descritores: Resumos estruturados. Recuperação da informação. Periódico científico eletrônico.

\footnotetext{
a Doutora em Ciência da Informação pela Universidade Estadual Paulista (UNESP). Professora do Departamento de Biblioteconomia da Universidade Federal do Espírito Santo (UFES). Email: paulaventuramorim@gmail.com.

b Doutora em Ciência da Informação pela Universidade Estadual Paulista (UNESP). Professora do Programa de Pós-Graduação em Ciência da Informação da Universidade Estadual de Londrina (UEL). E-mail: brigidacervantes@gmail.com.
} 


\section{INTRODUÇÃO}

A informação registrada desde sua geração até a sua recuperação e uso é o objeto de estudo da Ciência da Informação $(\mathrm{Cl})$ e está sistematizada nos processos de representação, descrição, organização, acesso, preservação, uso e recuperação de recursos informacionais em ambientes informacionais (BORKO, 1968; MARTÍNEZ RÍDER; RENDÓN ROJAS, 2004).

Para Mandl e Womser-Hacker (2001), a Ciência da Informação tem sua base nos processos de busca da informação feita a partir do uso de computador. Esse processo é apresentado por meio das tecnologias da informática e a informação é parte dos dados ou do saber do sistema computacional.

Nesse sentido, no que diz respeito à informação, a comunicação científica incorpora atividades relacionadas com a produção, disseminação e uso da informação, desde o momento da concepção da ideia de pesquisa até a aceitação da informação relativa aos resultados pelo cientista como constituinte do estoque universal de conhecimentos (GARVEY; GRIFFITH, 1979).

A partir dos anos de 1990, as Tecnologias de Informação e Comunicação (TIC) revolucionaram as formas de divulgação científica, pois, no ambiente Web, a capacidade de registrar informações aumentou vertiginosamente, porque é um ambiente onde todas as formas de comunicação se fundem. Assim, é necessário desenvolver processos que tornem o acesso à informação científica possível de maneira rápida, fácil, intuitiva e confiável.

O artigo científico é um trabalho acadêmico que apresenta resultados de pesquisa realizada de acordo com alguma metodologia e é publicado em periódicos ou anais de conferências. Segundo Sales (2013), o artigo é considerado fonte de conhecimento na comunicação formal da ciência.

Para Mueller (1999, p. 2), "O periódico em que o artigo científico é publicado se torna um ponto-chave em qualquer carreira científica, pois condiciona as chances da ocorrência de citações a esse artigo". Dessa maneira, para ser lido e citado, um artigo precisa ser encontrado pelo leitor, o que é chancelado por Marcondes (2001, p. 61), visto que ele é enfático ao afirmar: “[...] a informação relevante para um dado problema precisa estar disponível no 
tempo certo. De nada adianta a informação existir, se quem dela necessita não sabe da sua existência, ou se ela não puder ser encontrada".

Nesse sentido, o processamento eficiente da informação disponível tornase a cada dia um importante fator, para que ela possa expressar o seu valor e, por conseguinte, ser acessada, utilizada e apropriada por quem dela necessite.

Atendendo a uma demanda em que a eficiência, a rapidez e a facilidade de recuperação da informação são condições sine qua non, periódicos científicos eletrônicos da área médica já publicam os resumos em formato estruturado, procedimento pouco comum na Ciência da Informação.

Neste momento, é importante ressaltar que o resumo representa a versão precisa, sintética e seletiva do texto do documento, portanto, nele devem estar os elementos de maior importância. Desse modo, é necessário que o resumo seja feito de maneira itemizada, em que o conjunto de elementos traduza claramente e com fidedignidade o conteúdo do artigo.

O conjunto de elementos, ou seja, o Perfil de Aplicação dos Metadados (PAM), proporcionará a eficiência na busca, localização, acesso e recuperação da informação. Portanto, é necessário que os dados e metadados sejam descritos e estejam estruturados a partir de uma composição eficiente da linguagem nos sistemas de armazenamento de registros informacionais, que devem oferecer um maior grau de refinamento e granularidade.

Santos e Santana (2013, p. 206) definem que "[...] a granularidade de um conjunto de dados está vinculada ao número de atributos que o compõe a diversidade de seus conteúdos". Para os autores supracitados, "Quanto maiores as possibilidades de obter subconjuntos de dados, maiores são as possibilidades de tratamento e de elaboração de resultados". Nesse sentido, quanto maiores as possibilidades, maior o detalhamento disponível. (WANG; WU, 2003).

Diante desse contexto, a elaboração do resumo estruturado necessita da padronização e de diretrizes para o preenchimento dos elementos, ou seja, os resumos devem ser preparados cuidadosamente.

A partir do exposto, em consonância com os autores Mulrow, Thacker e Pugh, 1988; Endres-Niggemeyer, 1998; Sollaci e Pereira, 2004, o problema de pesquisa é contextualizado pela necessidade de que as publicações científicas 
eletrônicas publiquem artigos que contenham resumos estruturados, os quais apresentem em itens os elementos que descrevam o objetivo, a metodologia, os resultados e a conclusão. Assim, o resumo estruturado permitirá ao leitor localizar no artigo científico o que necessita com maior objetividade e rapidez, chancelando a importância dos registros do conhecimento e os processos de sistematização da informação para sua busca e recuperação. Afinal, essa preocupação, desde os primórdios, acompanha o profissional que tem a informação como seu objeto de trabalho, ou seja, o aprimoramento das técnicas para seu tratamento, no intuito de associá-la ao uso de alguma tecnologia, o que torna esse processo cada vez mais ágil, fácil e intuitivo.

Esta pesquisa tem como fio condutor as transformações da sociedade, a evolução das tecnologias de informática e o desenvolvimento das TIC. O desenvolvimento das TIC atingem dimensões inimagináveis quando integram os modos de comunicação da informação, oportunizando multiplicidade de ambientes informacionais.

Nesse cenário, é primordial que esses ambientes propiciem, de maneira fácil, rápida e consistente, os processos de busca, recuperação, acesso, difusão e aquisição da informação. Os artigos científicos são recursos para a comunicação formal da ciência. Para tanto, faz-se necessário efetivar processos que viabilizem a encontrabilidade e o acesso a eles.

\section{RESUMOS NOS ARTIGOS CIENTÍFICOS}

Ainda que a origem do artigo científico date de 1665 e, a partir de sua criação, terem acontecido muitas modificações, a descrição do método foi desenvolvida no decorrer da metade do século XIX. Já a estrutura formal foi convencionada de Introdução, Metodologia, Resultados e Discussão (IMRAD) no século XX (SOLLACI; PEREIRA, 2004).

Lancaster (2004, p. 100) tem a seguinte definição para resumo: "O resumo é uma forma sucinta, porém exata, do conteúdo de um documento". Na mesma linha de pensamento, Endres-Niggemeyer (1998, p. 132) afirma que "O resumo é um texto breve e coerente que deve informar o usuário sobre o conhecimento essencial transmitido por um documento". 
Dessa maneira, o resumo tem como principal objetivo apresentar ao leitor uma visão geral do que trata a investigação. A Norma Brasileira (NBR) oㅜ 6028:2003 estabelece os requisitos para a redação e apresentação de resumos. Essa norma foi criada no ano de 1990 e revista pela Associação Brasileira de Normas Técnicas em novembro de 2003. Segundo a definição da norma, o resumo deve ser a apresentação concisa dos pontos relevantes de um documento.

Em relação aos resumos, a ABNT - NBR 6028 (2003, p.1) indica que existem três tipos:

Resumo crítico: Redigido por especialistas com análise crítica de um documento. Resumo indicativo: Indica apenas os pontos principais do documento, não apresentando dados qualitativos etc. De modo geral, não dispensa a consulta ao original. Resumo informativo: Informa ao leitor finalidades, metodologia, resultados e conclusões do documento, de tal forma que possa inclusive, dispensar a consulta ao original.

Ainda que a NBR nos apresente três tipos de resumos, nos artigos científicos, a modalidade utilizada geralmente é o resumo informativo, o qual se constitui o nosso objeto de estudo.

Importante ressaltar que, para a decisão de leitura de um artigo científico, o título e o resumo são elementos essenciais, ou seja, se esses elementos fizerem sentido para as necessidades do leitor, este poderá se interessar pela pesquisa. Se o título chama a atenção do leitor, porém o resumo não é adequado, o leitor possivelmente não irá concluir a leitura do texto.

Lancaster (2004), ao discorrer sobre os resumos, afirma que, entre suas finalidades, a mais importante talvez seja que os resumos ajudam o leitor na decisão de ler ou não o texto completo e, assim, poupam o tempo do leitor.

Posto isso, verificamos que o resumo se torna peça fundamental para a divulgação e consequente leitura do artigo. Possivelmente, é a partir de sua leitura que o leitor tomará a decisão de ler ou não o artigo completo.

O resumo indicativo deve informar o objetivo, a metodologia, os resultados e as conclusões do documento. Tais indicações estão bem próximas à estrutura formal do IMRAD já citada.

Quanto à forma de apresentação, os resumos podem ser classificados em estruturados e não estruturados e, ainda que o conteúdo de ambos seja similar, 
há diferenças na maneira como serão apresentados.

O resumo estruturado é formado de extratos (itens ou seções). Cada extrato é precedido por um subtítulo. Os termos usados são apresentados de modo itemizado e são padronizados pelos periódicos científicos conforme um exemplo da revista Informação\&Informação, que apresenta 11 diretrizes para autores para a submissão de seus trabalhos. Assim, em sua diretriz número cinco, especifica: "5. Resumo estruturado, entre 150 e 250 palavras, escrito em português, inglês e espanhol, deve conter os seguintes itens: introdução, objetivo, metodologia, resultados e conclusões".

Segundo o Dicionário crítico para redação científica (2013, p. 173-174), o Resumo estruturado,

Como o nome sugere, é aquele que se apresenta em tópicos que correspondem à própria estrutura do artigo na íntegra. $\mathrm{O}$ resumo estruturado geralmente tem: objetivo, métodos resultados e conclusões. Em algumas revistas admite-se uma frase introdutória no tópico do objetivo ou o resumo inclui um tópico introdutório (background). O tópico e quantidades de itens variam, mas sempre aparecem destacados.

Ao discorrer sobre as diferenças entre resumo estruturado e resumo narrativo, o dicionário supracitado pontua que:

[...] o resumo narrativo cujo formato é livre permite que o autor descreva e justifique o que foi feito, mas não aonde se chegou com a pesquisa, obrigando o leitor ao ler o artigo descobrir a que conclusões chegaram. $O$ resumo estruturado obrigatoriamente inclui a conclusão da pesquisa. É mais informativo para o leitor (VOLPATO et al., 2013, p. 174).

A partir do exposto, podemos verificar que o leitor terá mais informações no resumo estruturado, o que lhe trará subsídios na decisão de utilizar ou não o artigo. Neste momento, é importante reforçar que o resumo poderá se configurar em uma única parte do artigo que o periódico tornará acessível para os mecanismos de busca.

A área da saúde foi pioneira em verificar a relevância dos resumos estruturados. Após pesquisas, verificamos que autores e leitores consideravam os resumos estruturados mais úteis que os tradicionais, o que levou o Grupo de Trabalho Ad Hoc à Avaliação Crítica da Literatura Médica e, no ano de 1987, a propor diretrizes para resumos informativos em sete títulos da área (GUIMARÃES, 2006). 
Nesse contexto, Mulrow, Thacker e Pugh (1988, p. 613. tradução nossa) apresentaram seis diretrizes para a elaboração de resumos informativos:

1.0 resumo deve começar com uma declaração precisa do objetivo principal da revisão. 2. As fontes de dados para a revisão devem ser resumidas de forma sucinta. 3. Os critérios utilizados para selecionar os estudos nas fontes de dados e o método pelo qual esses critérios foram aplicados devem ser especificados. 4. As diretrizes usadas para abstrair dados e avaliar a qualidade de dados devem ser descritas. 5. Os principais resultados da revisão e os métodos utilizados para obter esses resultados devem ser declarados. 6 . Conclusões e possíveis aplicações dos resultados devem ser declaradas de forma clara e sucinta.

O resumo estruturado descreve o estudo ao utilizar-se de títulos de conteúdo, fornecendo, dessa maneira, um conteúdo informativo relevante (GUIMARÃES, 2006; PEREIRA, 2013).

Ao discorrerem sobre a importância do resumo, Mensh e Kording (2017) afirmam que, para a maioria dos leitores, a única parte do artigo que será lida será o resumo, assim ele deverá transmitir efetivamente toda a mensagem de maneira eficaz. Os autores afirmam que ter uma estrutura bem delimitada ajudará a evitar um erro comum dos resumos, por exemplo, expor os resultados antes que os leitores estejam prontos para entendê-los.

O International Committee of Medical Journal Editors, em sua página ${ }^{1}$ de recomendações para a preparação do envio do manuscrito no que diz respeito ao resumo, traz a seguinte orientação:

Como os resumos são a única parte substantiva do artigo indexada em muitos bancos de dados eletrônicos e a única parte que muitos leitores leem, os autores precisam garantir que reflitam com precisão o conteúdo do artigo [...]. Os autores precisam preparar seus resumos estruturados no formato especificado pela revista que escolheram.

Alguns periódicos da Ciência da Informação, no Brasil, estratificados com Qualis de $A 1$ a B5, orientam ou fazem a exigência de resumos estruturados a autores quando realizam a submissão de seus artigos, ou seja, apresentam regras bem definidas para que os resumos sejam elaborados de modo estruturado. Para tanto, indicam aos autores os elementos que compõem o

1 Disponível em: http://www.icmje.org/recommendations/browse/manuscriptpreparation/preparing-for-submission.html\#b 
resumo estruturado. Outros periódicos sugerem que o resumo seja estruturado, porém não é uma exigência.

\section{SISTEMA DE GERENCIAMENTO DOS PERIÓDICOS CIENTÍFICOS}

Para o gerenciamento de periódicos científicos e publicação de revistas eletrônicas, foi especificamente desenvolvido o software de código aberto Software Open Journal System (OJS), pelo Public Knowledge Project (PKP), da University of British Columbia e da Simon Fraser University Library.

O software teve como foco atender à necessidade de criação e de gestão de uma revista eletrônica que se inserisse no Movimento Acesso Livre à Informação Científica (MÁRDERO ARELLANO, 2005). No Brasil, a iniciativa da disponibilização de periódicos partiu do Instituto Brasileiro de Informação em Ciência e Tecnologia (IBICT) que, no ano de 2003, constituiu um grupo de trabalho para tradução e estudo do software OJS, com o intuito de implantá-lo nos periódicos da área Ciência da Informação.

O OJS foi projetado para reduzir o tempo e a energia despendidos às funções administrativas relacionadas com a administração de um periódico, melhorando a manutenção dos registros e a eficiência dos processos editoriais. Para tanto, oferece infraestrutura técnica para a apresentação on-line dos artigos dos periódicos e dos fluxos de trabalho da gestão editorial.

Conforme o perfil apontado para as designações de funções no OJS, podemos verificar a importância do autor para a revista, pois, de acordo com Brito et al. (2018, p. 22), "[...] o autor é o usuário mais importante, visto que é ele quem fornece o conteúdo da revista". É a partir do resumo fornecido que o leitor tomará a decisão de ler ou não o artigo e utilizá-lo em seus trabalhos e pesquisa. Já o autor depende da citação do seu artigo publicado, o que é parte de uma cadeia que, consequentemente, aumentará o fator de impacto da revista, o índice h e o Qualis da publicação.

Como o trabalho em tela tem seu foco na representação visando a recuperação da informação, ou seja, a recuperação dos artigos publicados pelos periódicos eletrônicos qualificados, nosso interesse está, particularmente, no item "Submissão é feita on-line pelos autores", pois é na submissão que serão 
gerados os metadados, os quais ampliarão ou não o acesso pelos leitores aos conteúdos informacionais dos artigos publicados.

Com a consolidação da Web, na comunicação científica é real a necessidade de ampliação dos metadados para os atributos que compõem o resumo estruturado, o que resultará no aumento de possibilidades de acesso aos artigos publicados nas revistas.

No cenário em que a Ciência da Informação tem como características capitais organizar, representar e recuperar informações, há uma tendência no ambiente digital para que essas três práticas sejam feitas em uma plataforma, como acontece com os periódicos científicos.

Nesse sentido, o uso das tecnologias da informática tem sido periodicamente aplicado visando o aperfeiçoamento da área, porém é o processo de representação da informação que explicita características para a identificação, a padronização, a qualidade e a integridade dos dados, fazendo com que os metadados assumam o papel fundamental para que o usuário possa identificar, localizar, recuperar e acessar a informação (ARAKAKI, 2019).

Segundo a National Information Standards Organization (NISO) (2004, p.1), "Metadados são informações estruturadas que descrevem, explicam, localizam e, ainda, facilitam a recuperação, o uso e o gerenciamento de recursos de informação".

O nosso objetivo é propor um perfil de aplicação de metadados descritivos que "[...] apresentam informações sobre o conteúdo de um recurso, o que propiciará que eles sejam encontrados ou compreendidos" (NATIONAL INFORMATION STANDARDS ORGANIZATION, 2004).

Joudrey, Taylor e Wisser (2018, p. 184) afirmam que eles "Descrevem as características identificadoras e os contextos intelectuais dos recursos informacionais para fins de descoberta, identificação, seleção, aquisição, contexto e compreensão".

\section{PERFIL DE APLICAÇÃO DE METADADOS}

A interoperabilidade semântica é definida pela European Comission como: "[...] significado dos elementos de dados e os relacionamentos entre eles" 
(EUROPEAN COMISSION, 2010, p. 23). Para que seja possível a interoperabilidade semântica, faz-se necessária a utilização de um perfil de aplicação de metadados (PAM). Este perfil possibilitará que os elementos de dados possuam o mesmo significado e, dessa maneira, as partes se comuniquem (COYLE; BAKER, 2009; HEERY; PATEL, 2000).

A NISO (2007) apontou as especificações indicando como os esquemas de metadados se combinam e podem descrever recursos informacionais. Também explicita quais são e como os elementos de metadados são adotados na descrição.

Malta (2014, p. 227) afirma que o PAM se configura como "[...] um constructo genérico para desenhar registros de metadados que satisfaçam necessidades específicas das aplicações, proporcionando interoperabilidade semântica em outras aplicações".

Heery e Patel (2000, p. 1, tradução nossa) esclarecem:

Resourse Discovery Framewark (RDF) forneceu a tecnologia habilitadora para a combinação de elementos individuais a partir de uma variedade de esquemas diferentes, permitindo, assim, aos implementadores escolherem, identificarem os elementos que são mais adequados aos seus objetivos.

Os autores supracitados definem o padrão de aplicação de metadados como os elementos de dados extraídos de um ou mais esquemas de metadados procedentes de um ou mais namespaces, que são combinados por implementadores e otimizados para uma aplicação específica.

Para Nilsson, Baker e Johnston (2008, p. 3), criadores do documento Enquadramento de Singapura, um perfil de aplicação de metadados descreve "[...] o conjunto de diretrizes, regras de descrições e restrições usadas na criação de um conjunto específico de registros de metadados".

Andrade (2019, p. 245), ao discorrer sobre o Enquadramento de Singapura, diz:

Esse enquadramento apresenta a descrição de um conjunto de componentes necessários para o desenvolvimento de perfil de aplicação, assim como normas relacionadas com o modelo de domínio e os fundamentos da Web Semântica.

O Enquadramento de Singapura é um documento que visa a organização da comunidade de implementadores de PAM. Ao serem desenvolvidos padrões, 
a Estrutura de Singapura tem como objetivo a interoperabilidade máxima bem como a sua reutilização. Nesse contexto, são definidos um conjunto de componentes descritivos, necessários ou úteis para documentar um Perfil de Aplicação que descreve como esses padrões documentais se relacionam com modelos de domínio padrão.

Com a necessidade de uso de diferentes esquemas de metadados, estes devem ser definidos pelo modelo genérico para registros de metadados (DCAM). Segundo Heery e Patel (2000), é recomendado o uso do PAM.

\section{PROCEDIMENTOS METODOLÓGICOS}

Trata-se de uma análise exploratória com abordagem qualitativa a partir de levantamento bibliográfico. Para subsídio do embasamento teórico, foi realizada pesquisa na literatura da área da Ciência da Informação e da área médica.

Para chegarmos aos atributos aqui relacionados na área Ciência da Informação, a pesquisa documental foi feita a partir do levantamento dos periódicos da Ciência da Informação, qualificados pela Capes com extratos Qualis² A1, A2, B1, B2, B3, B4 e B5, quadriênio 2013 - 2016, ${ }^{3}$ no ano de 2018.

No levantamento dos periódicos, foram identificados 72 títulos na área da Cl apresentados nos Qualis: A1 - 16 periódicos; A2 - 9 periódicos; B1 - 18 periódicos; B2 - 9 periódicos; B3 - 5 periódicos; B4 - 1 periódico; e B5 - 14 periódicos. Desse total, em 13 (18,05\%) existem orientações para os autores considerarem o formato de resumo. Desses 13, em 7 (9,72\%) recomenda-se o uso de resumos estruturados. Já em 6 (8,33\%), o uso de resumo estruturado é compulsório.

Das seis revistas em que o resumo estruturado é compulsório, quatro utilizam o Software Open Journal Systems e duas adotam o sistema ScholarOne.

\footnotetext{
2 Sistema utilizado para classificar a produção científica dos programas de pós-graduação referente aos artigos publicados em periódicos científicos.

${ }^{3}$ Disponível em:

https://sucupira.capes.gov.br/sucupira/public/consultas/coleta/veiculoPublicacaoQualis/listaCo nsultaGeralPeriodicos.jsf
} 
É importante ressaltar que os periódicos nacionais fazem uso do software OJS.

Após o levantamento na área da Ciência da Informação, buscamos periódicos científicos da área médica, que já utiliza os resumos estruturados em seus artigos de periódicos, ou seja, já é uma norma em seus periódicos que autores apresentem nas submissões resumos itemizado. A pesquisa foi realizada em periódicos que apresentavam fator de impacto significativo. Assim, foram pesquisados artigos nas bases de dados Medical Literature Analysis and Retrieval System Online (MEDLINE) e Literatura Latino-Americana e do Caribe em Ciências da Saúde (LILACS) que se referiam a resumos estruturados. Para tanto, foram eleitos os 15 primeiros apresentados no ranking da ferramenta InCites Journal Citation Reports (JCR), 4 na categoria Medicina Geral. Posteriormente, verificamos a qualificação dos periódicos estratificados pela Capes no quadriênio 2013 - 2016.

A ferramenta JCR de recursos é publicada anualmente pela Thomson Reuters, antes conhecida como ISI, que disponibiliza dados de citação e publicação dos periódicos acadêmicos na área de Ciências e Ciências Sociais.

Verificamos que, de 15 periódicos, apenas 3 (20\%) não exigem que seus resumos sejam estruturados. Doze periódicos $(80 \%)$ são estratificados com Qualis A1 e dos outros três (20\%), um é qualis B2, um possui Qualis C e um não é indexado pela Capes.

A partir desse levantamento, foi possível detectar os elementos que não se repetem nos resumos da mesma área, ou seja, os elementos que são determinados pelo periódico. Diante dessa constatação, procedemos à identificação dos elementos que constam no resumo dos periódicos da Ciência da Informação e nos periódicos da área médica na categoria Medicina Geral. Nesse cenário, os elementos eram cotejados tendo em vista a criação de um modelo de dados.

No Quadro 1 - Comparativo dos elementos apresentados no resumo estruturado da Medicina Geral e Ciência da Informação -, é possível verificar os elementos do resumo estruturado das duas áreas.

\footnotetext{
${ }^{4}$ Disponível em:

https://jcrclarivate.ez43.periodicos.capes.gov.br/JCRJournalHomeAction.action\#
} 


\section{Quadro 1 - Comparativo dos elementos apresentados no resumo estruturado da Medicina Geral e da Ciência da Informação}

\begin{tabular}{|c|c|c|}
\hline Contextualização & Medicina Geral & Ciência da Informação \\
\hline & Background & Contextualização \\
\hline & Exposures & Introdução \\
\hline & Importance & $X x x x x$ \\
\hline \multicolumn{3}{|l|}{ Objetivo } \\
\hline & Objective & Objetivo \\
\hline \multicolumn{3}{|c|}{ Procedimentos metodológicos } \\
\hline \multirow{5}{*}{ Metodologia } & Methods & Método \\
\hline & Data collection & $X X X X X$ \\
\hline & Data analysis & Analysis \\
\hline & Design & Design \\
\hline & Setting & $x X X X X$ \\
\hline \multirow[b]{2}{*}{ Amostra } & Participants & $x x x x x$ \\
\hline & Criteria & Approach \\
\hline \multicolumn{3}{|l|}{ Resultados } \\
\hline & Findings & Resultados \\
\hline \multicolumn{3}{|l|}{ Conclusão } \\
\hline & Conclusion & Conclusões \\
\hline & Relevance & Considerações \\
\hline & $x x x x x$ & Originaly \\
\hline & $x x x x x$ & Value \\
\hline & $x X X X X$ & Lacuna \\
\hline & $x x x x x$ & Social implication \\
\hline \multicolumn{3}{|c|}{ Informações adicionais } \\
\hline & Funding & $x x x x x$ \\
\hline
\end{tabular}

Fonte: Dados da pesquisa fundamentados nos elementos do resumo estruturado solicitados pelos periódicos das áreas citadas.

${ }^{*}$ Os elementos foram mantidos no idioma apresentado.

A partir da retirada dos termos iguais de cada área, realizamos a categorização dos termos em: contextualização (background, contextualização, exposures, introdução e importance); objetivo (objective e objetivo); procedimentos metodológicos (metodologia: methods, métodos, data collection, data analysis, analysis, design, setting - amostra: participants, criteria, approach); resultados (findings e resultados); conclusão (conclusion, conclusões, relevance, considerações, original, value, lacuna, social implication); e informações adicionais (funding). Após o cotejamento, verificamos os elementos que corresponderiam à itemização do resumo estruturado.

Definido o modelo de dados, fizemos a tradução dos termos em propriedades de esquemas de metadados. Assim, foram utilizados os registros 
de metadados a partir da adoção do Linked Open Vocabulary (LOV). ${ }^{5}$

A definição da matriz de restrições foi elaborada a partir das orientações do método para o desenvolvimento de Dublin Core Application Profile Me4MAP. Conforme Malta e Baptista (2013), foram descritos classes, propriedades e esquemas de codificação, incluindo namespace, tipos de restrições e os valores dos strings.

Os esquemas de metadados utilizados foram: dcterms, fabio, owl, Isc, schema, log e rec.

\section{PERFIL DE APLICAÇÃO PARA RESUMOS ESTRUTURADOS (PARE)}

O desenvolvimento do PAM para resumos estruturados apresentados nos artigos científicos, aqui denominados de PARE, tem como objetivo descrever regras que atendam às necessidades dos usuários no que diz respeito à recuperação da informação, o que Lynch (1997, p. 32) descreve como "[...] uma comunidade em particular com requisitos de aplicação comum".

O PAM utilizado neste estudo é norteado pelos princípios do Enquadramento de Singapura adotados pela DCMI (DUBLIN CORE...,2014).

Nesse contexto, em conformidade com o método escolhido na primeira etapa, foram identificados e definidos os requisitos para o desenvolvimento do modelo de domínio e dos modelos de dados necessários.

Os requisitos funcionais são a base de avaliação do PAM em termos de consistência interna, o que demonstrará se esse PAM é apropriado ao uso em determinado contexto ou determinada comunidade. Dessa forma, os requisitos funcionais neste estudo possibilitarão o acesso aos artigos científicos a partir de atributos identificados e descritos no perfil de aplicação de metadados.

A Figura 1 demonstra a identificação modular para o esquema de descrição. Importante ressaltar que, ao ser proposto, o PAM, utilizando essa descrição, foi pensado nos princípios Linked Data.

\footnotetext{
${ }^{5}$ Disponível em: https://lov.linkeddata.es/dataset/lov/
} 
Figura 1 - Modelo dos Dados para Resumos Estruturados

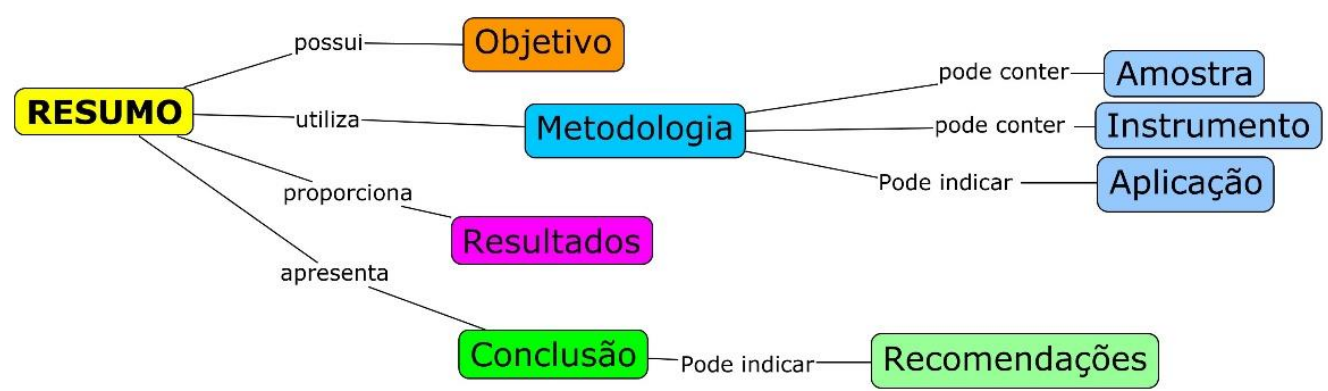

Fonte: Elaborada pelas autoras.

O modelo de dados foi definido após o cotejamento dos elementos, conforme o Quadro 1 - Comparativo dos elementos apresentados no resumo estruturado da Medicina Geral e Ciência da Informação -, ação que viabilizou o alinhamento de vocabulários, ou seja, foi feita a correspondência dos termos dos atributos em metadados, utilizando os registros de metadados. Neste estudo, foi utilizado o Linked Open Vocabulary (LOV) e o Dublin Core metadata.

Para melhor entendimento, foram usadas as seguintes definições para a construção da matriz de restrição:

Matriz de Restrições - definição detalhada de cada atributo ou entidade do Modelo de Domínio por meio de suas restrições, com a identificação dos esquemas de metadados, Vocabulary Encoding Scheme (VES) ou Syntax Encoding Scheme (SES) (MALTA, 2017);

Classe - alguma coisa usada principalmente para classificar ou categorizar outras coisas (W3C, 2004);

Atributo - dado significativo que pertence a uma classe. Geralmente contém valores que descrevem cada instância da classe (GLOSSARY OF INIFIED MODELLING..., 2017);

Propriedade - é um aspecto específico, relação utilizada para descrever um recurso;

Cardinalidade - descreve a lógica de relacionamento das informações, identificando a obrigatoriedade (pelo menos uma associação) (ANDRADE, 2019);

Uso - diz respeito ao uso de diferentes esquemas de metadados que atendem a necessidades específicas de aplicação (HEERY; PATEL, 2006); 
VES/SES - são esquemas de codificação, fornecem informações textuais ou regras de análise que auxiliam na interpretação de um valor do termo. $O$ Vocabulary Enconding Schemes (VES) indica que o valor é um termo de um vocabulário controlado e, portanto, auxilia na conceituação do recurso (os valores não são literais); e os Syntax Enconding Scheemes (SES) indicam que o valor é uma sequência de caracteres formatados conforme uma notação formal (os valores são literais) (DUBLIN CORE METADATA INITIATIVE, 2012b).

Foram utilizados, para a construção da matriz de restrições, os esquemas de metadados: dcterms, schema e foaf, e as ontologias bibo e dbpedia-owl a partir da Interface do Language Open vocabular (LOV) ${ }^{6}$ e DCMI Metadata Terms. ${ }^{7}$

Como a pesquisa tem como foco o resumo estruturado, seus elementos apresentam as seguintes definições:

- Amostra - uma parte de um todo maior (VOLPATO et al, 2013, p. 23);

- Aplicação - colocar em prática, aplicar o resultado da pesquisa em problemas específicos;

- Artigo científico - texto publicado em revista científica que apresenta conhecimento novo a partir de base empírica (VOLPATO et al., 2013);

- Conclusão - afirmativa teórica, com caráter de generalização, obtida a partir de uma base empírica (VOLPATO et al., 2013);

- Instrumento de coleta de dados - ferramentas específicas para coleta de dados (VOLPATO et al., 2013);

- Métodologia - processo sistemático de aquisição de conhecimento que segue uma série de passos independentes que, para efeitos didáticos, podem ser apresentados na seguinte ordem: definição do problema, formulação de hipóteses, raciocínio dedutivo, coleta de dados (CUNHA; CAVALCANTI, 2008);

- Objetivo - é o que se pretende atingir. Na pesquisa científica se refere ao que se quer atingir com a investigação (VOLPATO et al., 2013);

6 Disponível em: https://lov.linkeddata.es/dataset/lov/ . Acesso em: 7 ago. 2019

7 Disponível em: https://www.dublincore.org/specifications/dublin-core/dcmi-terms/\#http:// purl.org/dc/terms/rights 
- Recomendações - opinião do autor baseada no resultado de seu estudo, mas com a característica fundamental de que os achados sejam novos (originais) (VOLPATO et al., 2013);

- Resultados - fundamentação da conclusão (VOLPATO et al., 2013);

- Resumo estruturado - é aquele que se apresenta em tópicos que correspondem à própria estrutura do artigo na íntegra (VOLPATO et al., 2013).

A matriz de restrições foi elaborada tendo o resumo estruturado como uma instância do artigo científico. Para melhor detalhamento, a matriz de restrições é apresentada em dois quadros. O Quadro 2 define os namespaces e o Quadro 3, intitulado Matriz de restrição - resumo estruturado, define as propriedades da classe do resumo estruturado por ser uma instância do artigo científico.

Conforme o Quadro 2, podemos verificar os prefixos utilizados na matriz de restrições e o namespace correspondente ao prefixo.

\section{Quadro 2 - Definição dos namespaces utilizados}

\begin{tabular}{|l|l|}
\hline \multicolumn{1}{|c|}{ Prefixo } & \multicolumn{1}{c|}{ Namespace } \\
\hline dcterms & http://purl.org/dc/terms/ \\
\hline fabio & http://purl.org/spar/fabio/ \\
\hline owl & $<\mathrm{http}: / /$ www.w3.org/2002/07/owl\#> \\
\hline Isc & http://linkedscience.org//sc/ns\# \\
\hline schema & http://schema.org/ \\
\hline log & http://www.w3.org/2000/10/swap/log\# \\
\hline rec & http://purl.org/ontology/rec/core\# \\
\hline
\end{tabular}

Fonte: Elaborado pelas autoras e fundamentado no Language Open Vocabular (LOV).

A primeira linha dos quadros contempla:

a) descrição - o nome da classe; b) classe - o esquema de metadados ou o vocabulário e o termo utilizado; c) uso - uma descrição sucinta do que a classe representa.

A segunda linha diz respeito a cada propriedade do quadro de descrição definido:

a) atributo - etiqueta dada à propriedade; b) propriedade - esquema de metadados ou vocabulário respectivo ao termo utilizado (ex.: dcterms:title); c) cardinalidade - o número de vezes que a propriedade pode ser repetida (ex.: 0-1*) 0 - Não precisa ser apontada - precisa ser apontada pelo menos uma vez, $1^{*}$ é 
apontada mais de uma vez; d) namespace - identificador exclusivo; e) SES/VES - SES indica que o valor é um conjunto de caracteres formatado em conformidade com uma notação formal. VES Vocabulários controlados que auxiliam na conceituação do recurso (DUBLIN CORE METADATA INICIATIVE, 2012b); f) uso descrição concisa do que a propriedade representa.

A seguir, apresentamos o Quadro 3:

\section{Quadro 3 - Matriz de restrição - resumo estruturado}

\begin{tabular}{|c|c|c|c|c|c|}
\hline \multirow{2}{*}{$\begin{array}{l}\begin{array}{l}\text { Resumo } \\
\text { estruturad } \\
\text { o }\end{array} \\
\text { Atributo }\end{array}$} & \multicolumn{4}{|c|}{ Classe: fabio:Abstract } & \multirow{2}{*}{$\begin{array}{l}\text { Uso: Representação concisa de } \\
\text { um conteúdo apresentado em } \\
\text { tópicos que correspondem à } \\
\text { própria estrutura do artigo } \\
\text { Uso }\end{array}$} \\
\hline & $\begin{array}{c}\text { Propriedad } \\
\mathrm{e}\end{array}$ & $\begin{array}{l}\text { Cardinali } \\
\text { dade }\end{array}$ & Namespace & $\begin{array}{l}\text { SES/ } \\
\text { VES }\end{array}$ & \\
\hline Objetivo & $\begin{array}{l}\text { dbpedia- } \\
\text { owl:project } \\
\underline{\text { Objective }}\end{array}$ & 1 & $\begin{array}{l}\text { http://dbpedia.org/ontology } \\
\text { projectObjective }\end{array}$ & $\begin{array}{l}------ \\
\end{array}$ & $\begin{array}{l}\text { Define o objetivo da } \\
\text { pesquisa/estudo }\end{array}$ \\
\hline $\begin{array}{l}\text { Metodologi } \\
\text { a }\end{array}$ & $\begin{array}{l}\text { Isc:method } \\
\text { Used }\end{array}$ & $1^{*}$ & $\begin{array}{l}\text { http://linkedscience.org//sc } \\
\text { Ins\#methodUsed }\end{array}$ & $\begin{array}{l}------ \\
\end{array}$ & Método usado em uma pesquisa \\
\hline Resultado & $\begin{array}{l}\text { schema:res } \\
\text { ult }\end{array}$ & 1 & http://schema.org/result & $\begin{array}{ll}------ \\
\end{array}$ & O resultado produzido na ação \\
\hline Conclusão & $\begin{array}{l}\text { log:conclusi } \\
\text { on }\end{array}$ & 1 & $\begin{array}{l}\text { http://www.w3.org/2000/10 } \\
\text { /swap/log\#conclusion }\end{array}$ & ------ & Conclusão \\
\hline
\end{tabular}

Fonte: Elaborado pelas autoras.

A matriz de restrição foi desenvolvida visando ampliar as propriedades identificadas no esquema de metadados apresentado pelo Open Journal Systems. A primeira linha é composta pela descrição da classe do resumo estruturado:

Classe: $\mathrm{FaBio}^{8}$ - ontologia alinhada ao FRBR, com registro e publicação na Web Semântica e que faz parte do APAR (conjunto de ontologias de publicação e referência semântica);

Uso: este elemento descreve que o perfil será usado para a representação concisa de um conteúdo. No caso, o resumo estruturado será apresentado em tópicos que correspondem à própria estrutura do artigo.

Abaixo da primeira linha, a primeira coluna apresenta os atributos do padrão propostos a partir do alinhamento de vocabulário (Quadro 3). De acordo com as recomendações do ME4MAP, fazem parte deste PAM os atributos:

\footnotetext{
${ }^{8}$ Disponível em: https://lov.linkeddata.es/dataset/lov/vocabs/fabio
} 
objetivo, métodos de pesquisa, amostra, instrumento de coleta de dados, resultado, conclusão, recomendações e aplicação.

A coluna propriedades indica o esquema de metadados ou vocabulário respectivo ao termo escolhido que, nesse PAM, são: projectObjective, Isc:methodUsed, schema:sampleType, medred:hasInstruments, schema:result, log:conclusion, rec:recommends, schema:audience.

O modelo estabelece relações entre as propriedades que representam o resumo estruturado como instância do artigo científico. Tais relações dizem respeito à lógica dos relacionamentos das informações. Assim, indicam a obrigatoriedade, ou seja, deve ter pelo menos uma associação e/ou a cardinalidade (quantidade de instâncias de um objeto) (ANDRADE, 2019).

O atributo objetivo (1) deverá ser único no resumo estruturado. Já a metodologia poderá constar mais de uma $\left(1^{*}\right)$. O resultado e a conclusão serão únicos também (1). O namespace é apontado em todos os atributos.

O SES/VES não está especificado, visto que não foi encontrado em vocabulários controlados ou em formatos específicos.

Como nossa proposta é para um Perfil de Aplicação para Resumos Estruturados, sugerimos o modelo descrito na Figura 3 para o software Open Journal System.

A coluna uso na Figura 3 diz respeito a uma definição do atributo, ou seja, como ele deverá ser utilizado. Assim, o atributo objetivo deverá constar uma vez (cardinalidade) e deverá definir o objetivo da pesquisa/estudo. Obrigatoriamente, o autor deverá apontar a metodologia que foi utilizada na pesquisa, o(s) resultado(s) produzido(s) e a conclusão a que chegou a partir da pesquisa/estudo.

A matriz de restrição nos chancela a proposição do perfil de aplicação, conforme demonstrado na Figura 3: 


\section{Figura 3 - Perfil de Aplicação para Resumos Estruturados (PARE)}
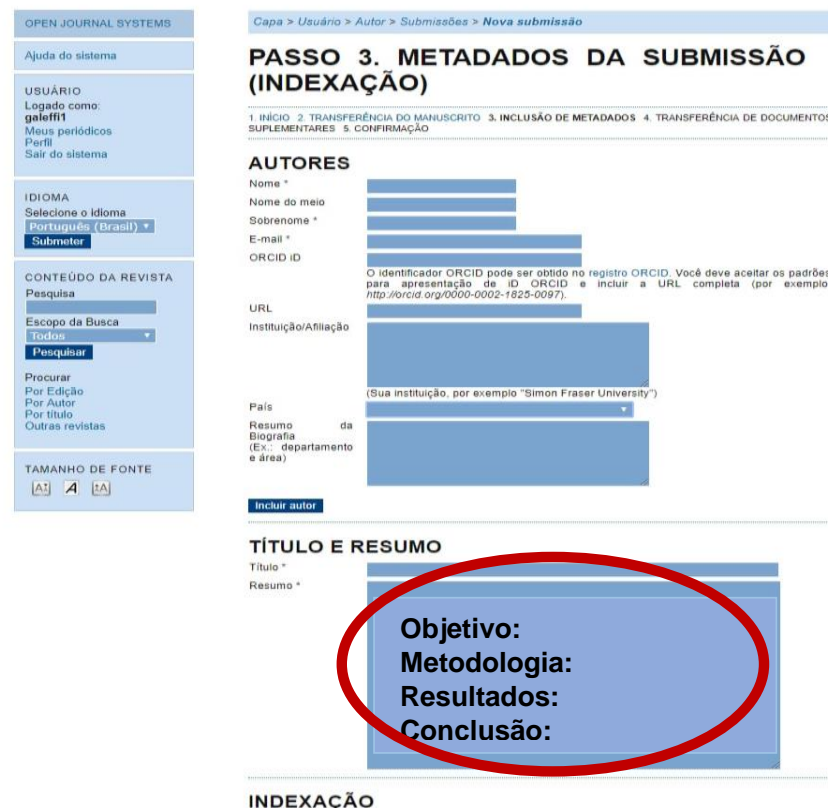

Fonte: Elaborada pelas autoras.

A partir da proposta do PARE, os autores, ao submeterem seus artigos a periódicos científicos, preencherão em itens os atributos estruturados - Objetivo, Metodologia, Resultados e Conclusão.

Em consonância com a proposta desta pesquisa, poderão ser ampliados os elementos de busca da informação na página disponibilizada a leitores de periódicos, que poderão satisfazer suas necessidades informacionais, ampliando os espaços de busca. Desta forma, para a busca, além de apenas por edição, por autor, por títulos e por outras revistas, também poderão utilizar as seguintes entradas: por objetivo, por metodologia e por resultados.

Buscando demonstrar a maneira como os resumos estruturados podem facilitar o entendimento do leitor, a seguir, trazemos dois resumos publicados em periódicos científicos da área e, ao lado, exibimos a proposta de resumos elaborados de maneira estruturada. No Quadro 4, apresentamos os resumos publicados e os resumos estruturados do artigo de Arraiza, Gonçalez e Vidotti (2019) (1) e Simionato et al. (2018) (2).

\section{Quadro 4 - Apresentação das Diretrizes}


pesquisa científica como forma para compartilhar os dados de pesquisa. Após analisar as publicações ampliadas e as abordagens presentes na literatura, descrevem-se as iniciativas atuais para arquivamento em repositórios de objetos de pesquisa. Como ponto principal do trabalho combinam-se os requerimentos gerais para repositórios digitais confiáveis da Research Library Group (RLG) e Online Computer Library Centre (OCLC), as pautas da norma ISO 16363:2012 e os requerimentos para sistemas de informação que trabalhem com publicações ampliadas. Como resultado propõem-se recomendações e formas de execução para a integração de publicações ampliadas em repositórios digitais confiáveis. Estas recomendações podem servir como base para futuros desenvolvedores de repositórios que queiram construir um marco de trabalho confiáve para este tipo de publicações

\subsection{RESUMO PUBLICADO (b)}

Objetivo: Estabelecer recomendações para a integração de publicações ampliadas em repositórios digitais confiáveis. As publicações ampliadas se configuram como objetos digitais compostos e estão adquirindo relevância na pesquisa científica como forma de compartilhar os dados de pesquisa. Método: Utiliza uma pesquisa qualitativa com caráter exploratório que analisa as publicações ampliadas e as abordagens presentes na literatura, bem como iniciativas atuais para arquivamento em repositórios de objetos de pesquisa. Além da análise, combinam-se os requerimentos gerais para repositórios digitais confiáveis da Research Library Group (RLG) e Online Computer Library Centre (OCLC), as pautas da norma ISO 16363:2012 e os requerimentos para sistemas de informação que trabalhem com publicações ampliadas. Resultado: Propõe-se uma série de recomendações e formas de execução para a integração de publicações ampliadas em repositórios digitais confiáveis. Conclusões: Essas recomendações podem servir como base para futuros desenvolvedores de repositórios que queiram construir um marco de trabalho confiável para tal tipo de publicações.

\section{2 - RESUMO PUBLICADO}

Como proponente da WebSemântica e dos princípios Linked Data, a iniciativa Linking Open Data oferece uma enorme proporção de dados de audiovisuais que podem auxiliar nas buscas e na recuperação de informações mais precisas. Nesse cenário, o objetivo é explorar os possíveis relacionamentos de bases de dados de audiovisuais no Linking Open Data, no intuito de apresentar o potencial dessa iniciativa para usuários que buscam fontes de informação detalhadas sobre os audiovisuais. Utilizou-se uma pesquisa de natureza qualitativa, com caráter exploratório e aplicado, baseada na literatura científica dos temas Linked Data, Web Semântica e audiovisual. Posteriormente, foram consultadas as bases DBpedia e LMDB com o uso do protocolo SPARQL. Considera-se que os conjuntos de dados disponíveis no Linking Open Data não só podem auxiliar na ligação entre informações sobre recursos audiovisuais, como também, podem ser fonte para a construção de catálogos mais dinâmicos, reduzindo o retrabalho durante o processo de descrição de recursos informacionais. confiáveis. As publicações ampliadas se configuram como objetos digitais compostos e estão adquirindo relevância na pesquisa científica como forma de compartilhar os dados.

Metodologia: Utiliza uma pesquisa qualitativa com caráter exploratório que analisa as publicações ampliadas e as abordagens presentes na literatura, bem como iniciativas atuais para arquivamento em repositórios de objetos de pesquisa. Além da análise, combinam-se os requerimentos gerais para repositórios digitais confiáveis da Research Library Group (RLG) e Online Computer Library Centre (OCLC), as pautas da norma ISO 16363:2012 e os requerimentos para sistemas de informação que trabalhem com publicações ampliadas.

Resultados: Propõe-se uma série de recomendações e formas de execução para a integração de publicações ampliadas em repositórios digitais confiáveis.

Conclusão: Essas recomendações podem servir como base para futuros desenvolvedores de repositórios que queiram construir um marco de trabalho confiável para tal tipo de publicações.

\section{2 - RESUMO ESTRUTURADO}

Objetivo: Explorar os possíveis relacionamentos de bases de dados de audiovisuais no Linking Open Data, no intuito de apresentar o potencial dessa iniciativa para usuários que buscam fontes de informação detalhadas sobre os audiovisuais

Metodologia: Pesquisa qualitativa com caráter exploratório; pesquisa aplicada. Consultadas as bases DBpedia e LMDB com o uso do protocolo SPARQL. A amostra contempla os filmes encenados pelo ator lan McKellen; filmes produzidos pelo estúdio Marvel Studios; e filme Priest of Love. Como instrumento de pesquisa, utilizou-se as bases de dados bibliográficas e conjunto de dados.

Resultados: Foram identificadas relações e as ligações que tanto a LMDB quanto a DBpedia fornecem a outras bases e plataformas da Web, como ao IMDb, site popular com informações sobre programas de televisão e filmes.

Conclusão: Considera-se que conjuntos de dados disponíveis no Linking Open Data não só podem auxiliar na ligação entre informações sobre recursos audiovisuais, como também podem ser fonte para a construção de catálogos mais dinâmicos, reduzindo o retrabalho durante o processo de descrição de recursos informacionais. Recomenda-se a construção de um ambiente informacional digital que permita aos usuários 


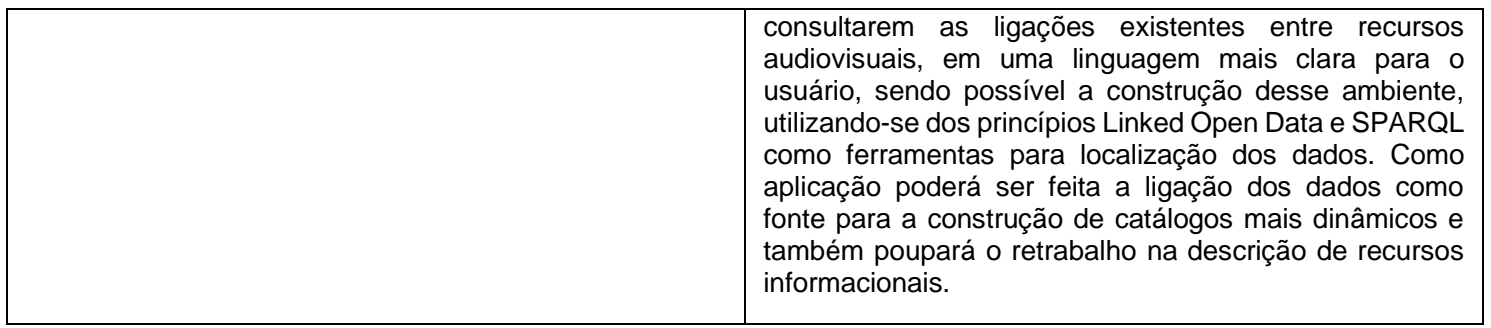

Fonte: Elaborado pelas autoras, a partir dos artigos de Arraiza, Gonçalez e Vidotti (2019) e Simionato et al. (2018).

Os dois resumos contêm até 250 palavras conforme solicitado pelos periódicos científicos da área de Ciência da Informação.

O artigo 1 de Arraiza, Gonçalez e Vidotti está disponibilizado em dois formatos pelo periódico: a) é apresentado quando o leitor visualiza somente o resumo e o formato; b) quando o leitor acessa o texto completo. São visíveis as diferenças de informação e de organização entre o resumo original e o resumo estruturado proposto. O resumo estruturado é mais longo, com 153 palavras contra 131 palavras do resumo (a), o que totaliza um aumento de $16,79 \%$.

No artigo de Simionato et al., o resumo estruturado também é mais longo - com 245 palavras, contra 155 palavras do resumo não estruturado, totalizando um aumento de $58,06 \%$, porém também é mais detalhado que o resumo original, visto que a amostra, os resultados e a aplicação são apresentados permitindo a ampliação de informação para o usuário. A conclusão também apresenta recomendações e aplicação da pesquisa.

Diante do exposto, a prática de resumo estruturado oferecerá aos autores uma estrutura para a organização e apresentação de maneira concisa dos resultados de suas pesquisas e aos leitores a possibilidade de identificar com eficiência pontos de interesse, ampliando os campos de recuperação, seleção e integração de informações, além de possibilitar que pesquisas bibliográficas remotas sejam mais precisas.

\section{CONSIDERAÇÕES FINAIS}

A facilidade de acesso à informação de maneira qualificada só será possível a partir da consistência no tratamento da informação. Na Ciência da Informação, o acesso e a recuperação de forma rápida, fácil e intuitiva têm sido 
uma preocupação recorrente e emergente frente ao aumento e à diversidade da informação desde os anos 90 do século XX.

Andrade (2019, p. 307) afirma que "A Web requer ferramentas e métodos para prover os utilizadores com informações relevantes em menos tempo e com menos esforço". Nesse sentido, os periódicos científicos, ao publicarem seus artigos na ambiência digital, podem contribuir significativamente com a minimização do tempo despendido por alunos e pesquisadores ao maximizar e objetivar a descoberta de recursos, por exemplo, considerando maior granularidade na descrição de artigos científicos espelhados em seus resumos.

Podemos inferir que a descrição completa e concisa do artigo é o resumo e ele se configura como o primeiro contato do leitor. Nesse contexto, sua importância é tal que, algumas vezes, o resumo é o único elemento recuperado ou revisado nas bases de dados.

Tendo como função ser claro, preciso e fiel ao texto completo, o resumo apresenta aos leitores um panorama sobre os principais pontos do artigo. Nessa perspectiva, em virtude das novas demandas de pesquisadores, a proposição do Perfil de Aplicação para Resumos Estruturados (PARE) se constituiu como o escopo deste trabalho.

A quantidade crescente de artigos científicos em todas as áreas do conhecimento humano aponta para a necessidade desses artigos serem cuidadosamente descritos. Como os resumos são o espelho das informações dos artigos, eles devem ser sistematicamente construídos para que os leitores possam identificar pontos relevantes de seu interesse que estejam cientificamente fundamentados.

No procedimento deste estudo, pudemos verificar que os resumos na área médica são estruturados. Entretanto, não há padronização, nem brasileira e nem internacional, dos elementos do resumo estruturado na área médica. Já na área de Ciência da Informação, a maioria dos periódicos não publicam seus resumos em formato estruturado. Poucos periódicos da Ciência da Informação requerem que o autor submeta um resumo estruturado e outros periódicos somente sugerem aos autores o fazerem. Ainda na área da $\mathrm{Cl}$, em algumas pesquisas, os autores apresentam ao leitor as duas formas de resumo, ou seja, depois de 
ter o artigo aprovado, deverá elaborar o seu resumo no formato estruturado. Assim, se o leitor fizer a pesquisa utilizando os motores de busca, o resumo se apresentará na forma convencional, mas se acessar o índice do periódico, ele virá acompanhado do texto no formato estruturado.

Observamos que estudos a respeito de resumos estruturados e sua implementação acontecem há algum tempo na área da Medicina Geral e da Ciência da Informação. Ainda que essa qualidade de resumo seja sugerida nas diretrizes para autores, poucos periódicos o fazem de forma compulsória e quase não se encontra pesquisa sobre o tema.

Trouxemos dois exemplos de resumos publicados de forma tradicional e estruturada para que fosse validada a percepção de que o formato de resumo estruturado facilita a tomada de decisão de ler ou não o artigo. Os resumos indicam que o uso de um padrão de metadados pelo software OJS possibilita encontrar a informação com menor esforço, ampliar as possibilidades de busca, de leitura e de citação, facilitando assim a revisão por pares.

Ao final deste estudo, as verificações realizadas nos conduzem a reflexões a respeito do papel dos bibliotecários frente às demandas em periódicos científicos para participar da modulação de dados, buscando a excelência na recuperação e no acesso à informação.

Recomendamos a adoção das diretrizes do modelo PARE para editores de periódicos científicos e comissão organizadora de eventos científicos.

\section{REFERÊNCIAS}

ARAKAKI, F. A. Metadados administrativos e a proveniência dos dados: modelo baseado na família PROV. 2019. 139 f. Tese (Doutorado em Ciência da Informação) - Faculdade de Filosofia e Ciências, Universidade Estadual Paulista, Marília, 2019.

ARRAIZA, P. M.; GONÇALEZ, P. R. V. A.; VIDOTTI, S. A. B. G. Recomendações para a integração de publicações ampliadas em repositórios digitais confiáveis. Encontros Bibli, Florianópolis, v. 24, n. 55, p. 1-23, maio 2019. Disponível em: https://periodicos.ufsc.br/index.php/eb/article/view/15182924.2019.e58556 Acesso em: 8 fev. 2020

ASSOCIAÇÃO BRASILEIRA DE NORMAS TECNICAS. NBR 6028: Informação e documentação: Resumo: Apresentação. Rio de Janeiro. 2003 
BORKO, H. Information science: what is it? American Documentation, v. 19, n. 1 , p. $3-5,1968$.

BRITO, R. F. Guia do usuário OJS 3. Brasília: IBICT, 2018. Disponível em: https://remgads.uerr.edu.br/public/site/Manual_OJS_IBICT_OJS_3_2018.pdf > Acesso em: 7 fev. 2020.

COORDENAÇÃO DE APERFEIÇOAMENTO DE PESSOAL DE NÍVEL SUPERIOR (CAPES). Disponível em: http://cotic.ufrrj.br/acesso-aos-periodicosda-capes/ Acesso em: 16 dez. 2020.

CUNHA, M. B.; CAVALCANTI, C. R. O. Dicionário de Biblioteconomia e Arquiovologia. Brasília. Briquet de Lemos, 2008.

DUBLIN CORE METADATA INITIATIVE. DCMI grammatical principles. 2012a. Disponível em: https://www.dublincore.org/specifications/dublincore/grammatical-principles/. Acesso em: 13 nov. 2019.

DUBLIN CORE METADATA INICIATIVE. DCMI metadata terms. 2012b. Disponível em: http://dublincore.org/documents/dcmi-terms Acesso em: 5 jan. 2020.

DUBLIN CORE METADATA INICIATIVE. The Singapore Framework for Dublin Core Aplication Profiles. Disponível em:

https://www.dublincore.org/specifications/dublin-core/singapore-framework/ Acesso em: 5 jan. 2020.

ENDRES-NIGGEMEYER, B. Summarizing information. Berlin. Springer, 1988.Disponível em: https://link.springer.com/content/pdf/10.1007\%2F978-3642-72025-3.pdf . Acesso em: 12 nov. 2019.

GARVEY, W. D., GRIFFITH, B. C. Communication and information process within scientific disciplines, empirical findings for psychology. In: GARVEY, W. D. Communication: the essence of science; facilitating information among librarians, scientists, engineers and students. Oxford: Pergamon, 1979. 332 p. Appendix A, p.127-147.

GUIMARÃES, C. A. Structured abstracts: narrative review. Acta Cir. Bras., São Paulo, v. 21, n. 4, p. 263-268, aug. 2006. Disponível em: http://www.scielo.br/scielo.php?script=sci_arttext\&pid=S010286502006000400014 Acesso em: 5 maio 2019.

HEERY, R.; PATEL, M. Application profiles: mixing and matching metadata schemas. Ariadne. Loughborough, ed. 25, 27-31, 2000. Disponível em: http://www.ariadne.ac.uk/issue/25/ Acesso em: 10 out. 2019.

INFORMAÇÃO \& INFORMAÇÃO. Londrina. UEL, 1996-. Disponível em http://www.uel.br/revistas/uel/index.php/informacao. Acesso em: 3 abr. 2020. 
JOUDREY, D. N.; TAYLOR, A. G.; WISSER, K. M. The organization of information. 4. ed. Santa Barbara, California: Libraries Unlimited, 2018. (Library and information science text series).

LANCASTER, F. W. Indexação e resumos: teoria e prática. 2. ed. Brasilia, Briquet de Lemos, 2004.

LYNCH, E.The Z39.50 Information Retrivial Standar. part I: A strategic View of the past, present and future. D-Lib Magazine, v. 11, n. 9. 1997.

MALTA, M. C.; BAPTISTA, A. A. The development process of a metadata application profile for the social and solidarity economy. In: MALTA, M. C.; BAPTISTA, A. A.; WALK, P. (org.). Developing metadata application profiles. Hershey: IGI Global, 2017.

MALTA, M. C.; BAPTISTA, A. A. Me4DCAP V0. 1: a method for the development of Dublin Core Application Profiles. Information Services \& Use, V. Guimarães - Portugal, v. 33, n. 2, p. 161-171, 2013.

MALTA, M. C. Contributo metodológico para o desenvolvimento de perfis de aplicação no contexto da Web Semãntica. 2014. 398 f. Tese (Doutorado em Tecnologias e Sistema de Informação) - Universidade do Minho, Braga, 2014.

MANDL, T.; WOMSER-HACKER, C. Curriculos da ciência da informação na Alemanha. 2001. Disponível em: https://www.researchgate.net/publication/242433122_Curriculos_da_Ciencia_d a_Informacao_na_Alemanha Acesso em: 5 fev. 2020.

MARCONDES, C. H. Representação e economia da informação. Ciência da Informação, Brasília, v. 31, n. 1, p. 61-70, jan./abr. 2001. Disponível em: https://www.scielo.br/pdf/ci/v30n1/a08v30n1 Acesso em: 15 jan. 2021

MÁRDERO ARELLANO, M. A.; SANTOS, R.; FONSECA, R. SEER:

Disseminação de um sistema eletrônico para editoração de revistas científicas no Brasil. Arquivística.net, Rio de Janeiro, v. 1, n. 2, p. 75-82, jul./dez. 2005. Disponível em: https://core.ac.uk/download/pdf/11889732.pdf. Acesso em: 21 jan. 2019.

MARTÍNEZ RÍDER, R. M.; RENDÓN ROJAS, M. A. Algunas propuestas latinoamericanas de objetos de estudio para la investigación bibliotecológica. Revista Ineramericana de Bibliotecología, Medellín, v. 17, n. 1, p. 13 - 44, 2004.

MENSH, B.; KORDING, K. Ten simple rules for structuring papers. PLoS Comput Biol, San Francisco, v. 13, n. 9, p. 1- 9, 2017. Disponível em: https://journals.plos.org/ploscompbiol/article/file?id=10.1371/journal.pcbi.10056 19\&type=printable. Acesso em: 2 out. 2019. 
MUELLER, S. P. M. O círculo vicioso que prende os periódicos nacionais. DataGramaZero: Revista da Ciência da Informação, dez.1999. Disponível em: https://repositorio.unb.br/handle/10482/985 . Acesso em 16 dez. 2020.

MULROW, C. D.; THACKER, S. B; PUGH, J. A. A proposal for more informative abstract of review articles. An Intern Med. Filadélfia, v. 108, p. $613-615$, 1988. Disponível em: https://www.ncbi.nlm.nih.gov/pubmed/3348568. Acesso em: 6 maio 2019.

NATIONAL INFORMATION STANDARDS ORGANIZATION. Understanding metadata: what is metadata, and what is it for? Baltimore: NISO, 2017. Disponível em:

https://groups.niso.org/apps/group_public/download.php/17446/Understanding \%20Metadata.pdf . Acesso em: 16 dez. 2020.

PEREIRA, M. G. O resumo de um artigo científico. Epidemiol. Serv. Saúde, Brasília, v. 22, n. 4, p. 707-708, dez. 2013. Disponível em: http://scielo.iec.gov.br/scielo.php?script=sci_arttext\&pid=S167949742013000400017\&lng=pt\&nrm=iso\&tlng=pt Acesso em: 13 dez. 2019

SALES, D. P. Critérios de avaliação da produção científica em ciências sociais aplicadas: inquirindo as bases de dados. 2013. 115 f. Dissertação (Mestrado em Ciência da Informação) - Escola de Comunicação e Artes, Universidade de São Paulo, São Paulo. Disponível em: https://www.teses.usp.br/teses/disponiveis/27/27151/tde-26052015-122438/ptbr.php. Acesso em: 16 dez. 2020.

SANTOS, P. L. V. A. C.; SANTANA, R. C. G. Dado e granularidade na perspectiva da informação e tecnologia: uma interpretação pela Ciência da Informação. Ciência da Informação, Brasília, v. 42, n. 2, p. 199-209, 2013. Disponível em: http://revista.ibict.br/ciinf/article/view/1382. Acesso em: 3 abr. 2020.

SIMIONATO, A. C.; CONEGLIAN, C. S.; GONÇALEZ, P. R. V. A.; SANTAREM SEGUNDO, J. E. Audiovisuais e Linked data: um estudo das bases DBpedia e LMDB. Em Questão, Porto Alegre, v. 24, n. 3, set./dez. 2018. Disponível em: https://seer.ufrgs.br/EmQuestao/article/view/78206 . Acesso em: 7 fev. 2020.

SOLLACI, L. B.; PEREIRA, M. G. A introdução, métodos, resultados e estrutura de discussão (IMRAD): uma pesquisa de cinquenta anos.J Med Libr Assoc. Rockville. v. 92, n. 3, p. 364-371, 2004. Disponível em: https://www.ncbi.nlm.nih.gov/pmc/articles/PMC442179/\#i0025-7338-092-030364-b1. Acesso em: 23 jun. 2019.

WANG, L.; WU, G. Attribute reduction and information granularity. Journal of Systemics, Cybernetics and Informatics. International Institute of Informatics and Cybernetics, v. 1, n.1, p. 32-37. 2003. Disponível em: https://pdfs.semanticscholar.org/0f35/c6ff5f790ad7431ccc810c6f55b0bf2e07a8. pdf. Acesso em: 3 abr. 2019. 
W3C. RDF Vocabulary description language 1.0: RDF Schema. 2004.

Disponível em: https://www.w3.org/TR/rdf-schema/. Acesso em: 23 jun. 2019.

\title{
APPLICATION PROFILE FOR STRUCTURED ABSTRACTS (APSA)
}

\begin{abstract}
Introduction: The abstract represents the precise, synthetic and selective version of the document's text, therefore, on him it must be the most important elements. Thus, it is necessary that the abstract is done in an itemized way, in which the set of elements clearly and reliably translates the content of the paper. Objective: To propose a metadata application profile for describing elements of a structured abstract of scientific articles published in electronic scientific journals. Methodology: Exploratory analysis with a qualitative approach. The research was conducted in the literature of the areas of General Medicine and of Information Science. In the medical area, which already uses structured abstracts, articles were searched in the Medical Literature Analysis and Retrieval System Online (MEDLINE) and Latin American and Caribbean Literature in Health Sciences (LILACS) databases. In the area of Information Science, documentary research was executed based on a survey of journals qualified by Capes, in the 20132016 quadrennium. The sample was composed of 72 titles in the area of Information Science and the first 15 titles presented in the InCites Journal Citation Reports JCR) and stratified by Capes. Results: The Proposition of the Application Profile for Structured Abstracts (PARE). Conclusion: The abstract must reflect the information contained in the articles. Additionally, the abstract should be systematically constructed for readers be able to: identify relevant points of interest that are scientifically based; have a structure that presents concisely the results of the research; and search through more accurate search engines.
\end{abstract}

Descriptors: Structured abstracts. Information retrieval. Electronic scientific journal.

\section{PERFIL DE APLICACIÓN PARA RESÚMENES ESTRUCTURADOS (PARE)}

\section{RESUMEN}

Introducción: El resumen representa la versión precisa, sintética y selectiva del texto del documento, por lo que los elementos de mayor importancia deben estar presentes en él. Por tanto, es necesario que el resumen se realice de forma desglosada, en la que el conjunto de elementos traduzca de forma clara y fiable el contenido del artículo. Objetivo: Proponer un perfil de aplicación de metadatos para la descripción de elementos del resumen estructurado en el ámbito de los artículos científicos publicados en revistas científicas electrónicas. Metodología: Análisis exploratorio con enfoque cualitativo. La investigación se realizó en la literatura del campo de la Medicina General y en el área de las Ciencias de la Información. En la literatura médica, que ya utiliza resúmenes estructurados, se buscaron artículos en las bases de datos Medical Literature Analysis and Retrieval System Online (MEDLINE) y Latin American and Caribbean Literature in Health Sciences (LILACS). En el área de Ciencias de la Información, la investigación documental se realizó a partir de una encuesta de revistas 
calificadas por Capes, en el cuatrienio 2013-2016, con una muestra de 72 títulos en el área de Ciencias de la Información y los primeros 15 títulos presentados en el ranking InCites Journal Citation Reports (JCR) y estratificado por Capes. Como instrumentos de investigación se utilizó: Extracto de Capes para revistas del área de Ciencias de la Información y la herramienta InCites Journal Citation Reports (JCR) para revistas del área de Medicina General. Resultados: Se presenta la Propuesta de Perfil de Aplicación para Resúmenes Estructurados (PARE). Conclusión: El resumen debe reflejar la información contenida en los artículos y debe ser construido de manera sistemática para que los lectores puedan: identificar puntos de interés relevantes que tengan una base científica; tener una estructura que presente los resultados de la investigación de manera concisa; y busque motores de búsqueda más precisos.

Descriptores: Resúmenes estructurados. Recuperación de información. Revista científica electrónica.

Recebido em: 15.02 .2021

Aceito em: 22.02.2021 